\title{
Physics as Final Opportunity to Prevent Harms Related to Theatricalization of Meteorology
}

\author{
Mbane Biouele César \\ Laboratory of Earth’s Atmosphere Physics, Department of Physics, University of Yaoundé I, Yaoundé, Cameroun \\ Email: cesar.mbane@yahoo.fr
}

Received November 28, 2013; revised December 25, 2013; accepted January 2, 2014

Copyright (C) 2014 Mbane Biouele César. This is an open access article distributed under the Creative Commons Attribution License, which permits unrestricted use, distribution, and reproduction in any medium, provided the original work is properly cited. In accordance of the Creative Commons Attribution License all Copyrights @ 2014 are reserved for SCIRP and the owner of the intellectual property Mbane Biouele César. All Copyright @ 2014 are guarded by law and by SCIRP as a guardian.

\section{ABSTRACT}

Using Mbane Biouele formula derived in 2009 on the troposphere thermoelastic properties leads to thermal and kinematic profiles of major atmospheric disturbances which clearly indicate that these terrible events for men should not be viewed with fatalism. This unexpected truth is unfortunately always obscured by media outlets of brilliant TV presenters or famous workshops panelists that focus attention on the excessively sensational meteorology (unfortunately folk and pernicious) instead of worrying about the seriousness that should characterize all interventions on the climate study or prediction. Good weather conditions, it is undeniable, facilitate an excellent running of almost all human activities like sports, transport, agricultural activities, celebrations of events, etc.... Far more serious, the advent of supercomputers and satellites could, if their valuable information is used solely for the theatricalization of weather events, trigger the decline of the scientific discipline of great public utility that is meteorology. Indeed, many meteorologists acquire very big head when they succeed in acquiring advanced equipment. Without prejudging what meteorology will become in the future, we hope that the work done in this article will remind each researchers that much remains to be done to promote climate studies. We remind quite emphatically that both hurricanes and cyclones have their weak-points (or talon d'Achilles in French) and thus, researchers should begin to think about "how to neutralize atmospheric disturbances that have both a large and a strong destructive power".

\section{KEYWORDS}

Mbane Biouele Formula Derived in 2009; Kinematic Profiles of Major Atmospheric Disturbances; Theatricalization of Meteorology; Cyclones Have Their Weak-Points or “Talon d'Achilles"

\section{Introduction}

The advent of supercomputers and satellites could, if their valuable information is used solely for the theatricalization of weather phenomena, trigger the decline of meteorology. Indeed, many meteorologists become proud to excess (similar to cinematograph films stars) from the time they work in structures with worldwide reputation. These celebrities of a different kind refuse any contradiction (including the most relevant) and unfortunately convey erroneous and inconsistent information during their numerous interventions in the workshops. Fortunately, physics appears today as final opportunity to prevent harms related to excessive theatricalization of meteorology. Fortuitously, the application of Mbane Biouele formula derived
2009 on troposphere thermoelastic properties [1-5] and its related corollaries lead to appropriate and unique profiles of major weather disturbances which show without any dough for us that both cyclones and tornadoes have their weak-point or talon d'Achilles and should not be viewed with fatalism. We hope that this unexpected reality will remind each researcher that much remains to be done to promote our knowledge of the atmosphere disturbances that have both a large and a strong destructive power.

\section{Mbane Biouele Formula on the Troposphere Thermoelastic Properties}

\subsection{Atmosphere Dynamics Concept of Air Particle}

Atmosphere Dynamics use a set of assumptions (previ- 
ously uncontested) to define the particle or parcel of air [6-8]. Especially:

1) Few exchanges on molecular scale: it is easy to follow quantity of air which preserves certain properties.

2) Quasi-static equilibrium: at any moment there is dynamic balance, i.e., the particle has the same pressure as its environment $\left(P=P_{\text {ext }}\right)$.

3) No thermal balance: heat transfers by conduction are very slow and neglected. One can have $T \neq T_{\text {ext }}$.

4) The horizontal sizes of the air particle can go from a few $\mathrm{cm}$ to $100 \mathrm{~km}$ according to the applications.

\subsection{Mbane Biouele Formula on the Troposphere Thermoelastic Properties}

Taking into account the fact that the atmosphere is mainly composed of dry air and water vapor, the Dalton's law connects the pressure $(P)$ with the partial pressure of dry air $\left(P_{a}\right)$ and saturated water vapor $\left(e_{w}\right)$

$$
P=P_{a}+e_{w}
$$

In deriving $(P)$ with respect to the temperature, one has

$$
\frac{\mathrm{d} P}{\mathrm{~d} T}=\left(\frac{\partial P}{\partial T}\right)_{V}+\left(\frac{\partial P}{\partial V}\right)_{T}\left(\frac{\mathrm{d} V}{\mathrm{~d} T}\right)
$$

According to the Quasi-static equilibrium (or dynamic balance) the pressure of the parcel of air must be the same as that of the ambient air, including during sudden local changes in phases by water contained in this parcel. Reality that all meteorologists restore through the following famous sentence: the phases change takes place at constant pressure. Thereby write Equation (3),

$$
\mathrm{d} P=0
$$

Equations (2) and (3) lead to the derivative of $V$ compared to $T$

$$
\frac{\mathrm{d} V}{\mathrm{~d} T}=-\frac{\left(\frac{\partial P}{\partial T}\right)_{V}}{\left(\frac{\partial P}{\partial V}\right)_{T}}
$$

Introducing the coefficient of thermal expansion of moist air at constant temperature

$$
\chi=-\frac{1}{P}\left(\frac{\partial P}{\partial V}\right)_{T}
$$

Then the Fundamental Relationship of Atmosphere Dynamic Balance:

$$
\frac{\mathrm{d} V}{\mathrm{~d} T}=\frac{1}{\chi} \bullet \frac{1}{P}\left(\frac{\partial P}{\partial T}\right)_{V}
$$

One can also write equation of Atmosphere Dynamic Balance in terms of partial pressures

$$
\frac{\mathrm{d} V}{\mathrm{~d} T}=\frac{1}{\chi} \bullet \frac{1}{P}\left[\left(\frac{\partial P_{a}}{\partial T}\right)_{V}+\left(\frac{\partial e_{w}}{\partial T}\right)_{V}\right]
$$

$\left(\frac{\partial P_{a}}{\partial T}\right)_{V}$ is negligible compared to $\left(\frac{\partial e_{w}}{\partial T}\right)_{V}$

Thereby write formula (8) named Mbane Biouele formula on troposphere thermoelastic properties

$$
\frac{\mathrm{d} V}{\mathrm{~d} T} \cong \frac{1}{\chi} \bullet \frac{1}{P}\left[\left(\frac{\partial e_{w}}{\partial T}\right)_{V}\right]
$$

\section{Formula (8) leads to a meteorological very useful} statement:

At any moment and throughout the atmosphere, one can use formula (8) and Clausius-Clapeyron slope of the equilibrium curves in the eT-diagram (Figure 1) to predict in which direction the air parcel will move (up or down) if its temperature increases or decreases. Table 1 or Figure 2 provides an overview of possible situations throughout the Troposphere.

\section{Evidence of Effectiveness of Mbane Biouele Formula (2009)}

The two equal level surfaces of water vapor and temperature rating respectively at $6.11 \mathrm{mb}$ and $0.0098^{\circ} \mathrm{C}$

\begin{tabular}{|c|c|c|c|}
\hline $\begin{array}{c}\text { Range of temperature } \\
\text { coupled with range of } \\
\text { humidity }\end{array}$ & $\begin{array}{l}T<0.0098^{\circ} \mathrm{C} \\
\boldsymbol{e}_{w}<6.11 \mathrm{mb}\end{array}$ & $\begin{array}{l}T<0.0098^{\circ} \mathrm{C} \\
\boldsymbol{e}_{w}>6.11 \mathrm{mb}\end{array}$ & $\begin{array}{l}T>0.0098^{\circ} \mathrm{C} \\
e_{w}>6.11 \mathrm{mb}\end{array}$ \\
\hline$\left(\frac{\partial e_{W}}{\partial T}\right)_{V}$ & + & - & + \\
\hline$\frac{\mathrm{d} V}{\mathrm{~d} T}$ & + & - & + \\
\hline
\end{tabular}

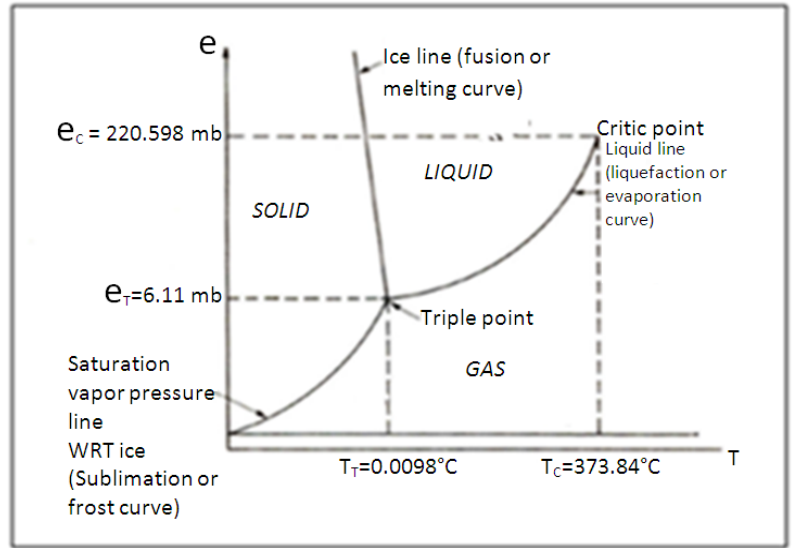

Figure 1. Saturation curves for water substance onto the $e T$-plane ( $e_{w T}$ and $T_{T}$ are triple-point coordinates): $e_{w T}=$ $6.11 \mathrm{mb} ; T_{T}=0.0098^{\circ} \mathrm{C}$.

Table 1. Changes in volume of the moist air particle depending on temperature within a specific range of temperature and humidity. 


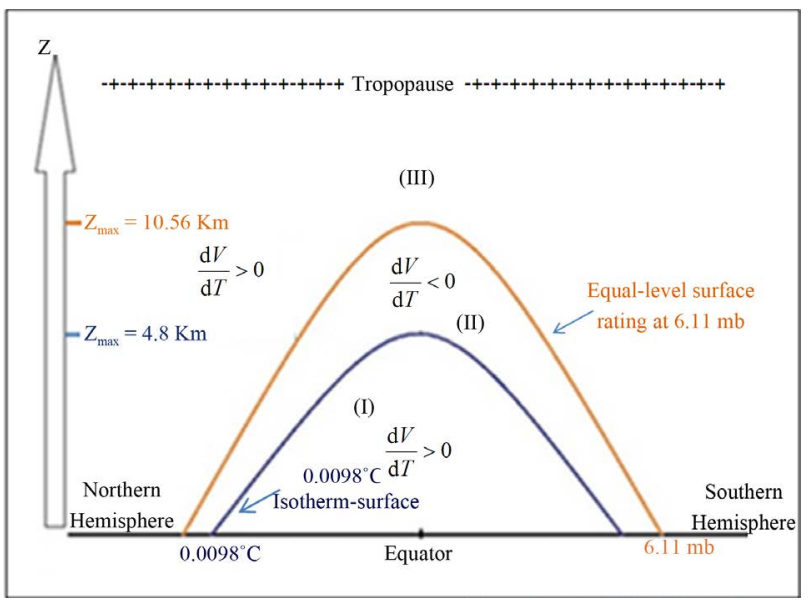

Figure 2. Troposphere specific regions depending on the manner in which $V$ changes with $T$ ( $V$ and $T$ are respectively volume and temperature of an air parcel): If $(\mathrm{dV} / \mathrm{d} T)>0$ the particle swells when its temperature increases (so it becomes lighter), if $(\mathrm{dV} / \mathrm{d} T)<0$ the particle shrinks when its temperature increases (so it becomes less light). $Z_{\max }$ are statistical value of observed maximum elevation of equal level surfaces of temperature and water vapor rating respectively at $0.0098^{\circ} \mathrm{C}$ and $6.11 \times 10^{-3}$ bars.

(Figure 2) separate clearly parts of the troposphere where ideal gas assumption can be applied without too distort reality to parts of the troposphere where this assumption is banned: hence appropriate and unique plots of thunderclouds profiles of wind and electric charges and earth's atmosphere tricellular circulation.

\subsection{Thunderclouds Winds' Profiles and Related Electrical Charges}

All natural meteorological phenomena included Hurricanes and Cyclones can be traced to the manner in which the energy from the sun is received over different parts of the earth. Since the troposphere is a medium in which mass motions are easily started, convection is found to be one of the chief ways in which heat is transferred there. This transfer may be accomplishing either by vertical or by horizontal motions. According to our results: warmer disturbances that occur in lower-troposphere are dissipated by a typical mass motion usually called Hurricanes (or tornadoes) while cooler disturbances that occur in mid-troposphere are dissipated by another typical mass motion called Cyclones. Knowing that Coriolis force act to west on updrafts, everyone can now understand why Hurricane and Cyclone move preferentially from East to West due to the localization in updrafts of their heat sources. Cyclones' heat source is made of huge and cooler fogs (those observed temperatures are less than $-45^{\circ} \mathrm{C}$ ) which can travel even increase (over hot oceans) in the troposphere while hurricanes' heat source is fixed on the Ground: that's why Cyclone lives and travels longer than Hurricane.

a) Schematic representations of Hurricanes and related cloud or electrical systems

Hurricanes appear (Figure 3(a)) as very high towers (from 0 to about $9 \mathrm{Km}$ ) consisting of three floors: warm updrafts occupying the first and third while warm downdrafts occupies the second floor. According to ground based observations, over-land hurricanes (or tornadoes) trigger thunderclouds whose base is thin compared to the peak which is very broad. Lower troposphere updrafts of Figure 3(a) can, in the same place, simultaneously trigger (due to wind-stress and adiabatic expansion) electrical positive charges and dark-cloud (Stratus) as suggested in Figure 3(b). The broadest peaks of the related clouds indicate the presence of the second floor warm downdrafts that prevent the progression of the first floor warm updrafts. Figure 3(a) is completely different to theatrical Figure 3(c) which contains many contradictions. e.g.: 1) Tornado or Cyclone eyes are known as a low pressure: the diagram says otherwise placing the eye at the center of divergence; 2) the veil of thick and very high white cloud (named Cumulonimbus) that envelops the black cloud formation is present nowhere in the earth's atmosphere (including on pictures taken on Tornadoes); 3) downdrafts generally do not create the clouds and cannot trigger tornadoes dark clouds as shown in the diagram.

b) Schematic representations of Cyclones and related cloud or electrical systems

Cyclones appear (Figure 4(a)) as very high towers (from 0 to about $14 \mathrm{Km}$ ) consisting of three floors: cooler downdrafts occupying $1^{\text {st }}$ and $3^{\text {rd }}$ while cooler updrafts occupy the $2^{\text {nd }}$ floor. There is good agreement between aircraft-based observations and related cyclones second floor updrafts convective clouds whose base has to be located above $\mathbf{0 . 0 0 9 8}{ }^{\circ} \mathbf{C}$ isotherm surface. Mid troposphere downdrafts of Figure 4(a) can, in the same place, simultaneously trigger (due to wind-stress and rapid-cooling of troposphere lower layers below their dew point temperature) electrical positive charges and darkcloud (Stratus) as suggested in Figure 4(b). View from the ground surface, the three cloud systems of Figure 4(b) appear to the observer as a single very high vertical extension cloud named Cumulonimbus by meteorologist. The same clouds system, seen from an airplane flying at 7000 meters, is as shown in Figure 4(b). i.e.: Low-altitudes stratus above which is placed a single cumulus whose base is broader than its top.

c) Schematic representations of Hurricane and Cyclone horizontal winds

Observed pressure near the eyes of Cyclones (or hurricane) is very low and concentrates a rapid decrease in a short distance so that the momentum of particles of air, 


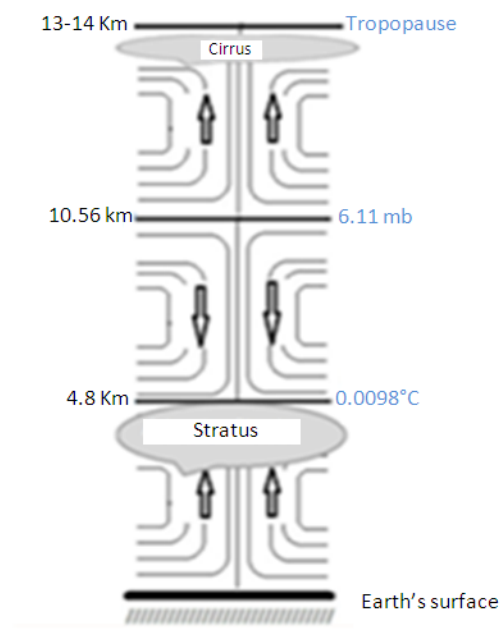

Vertical profile of Hurricane and related clouds system (Mean statistical values are in dark color while Thermodynamic values are in blue color)

(a)

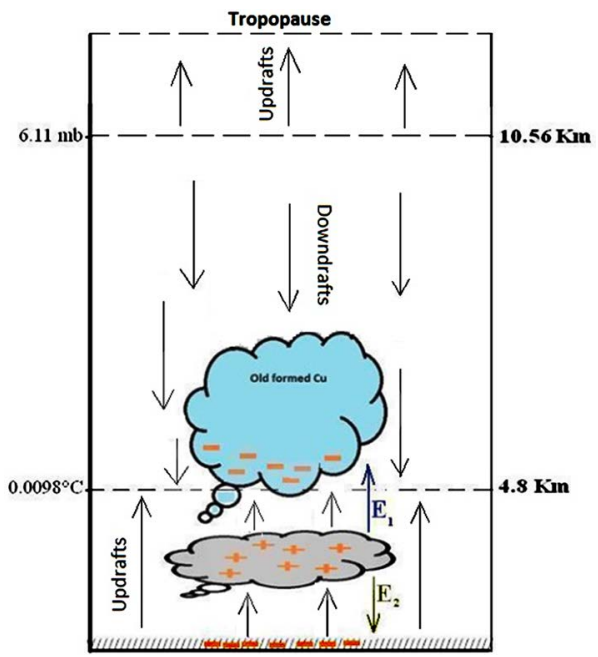

(b)

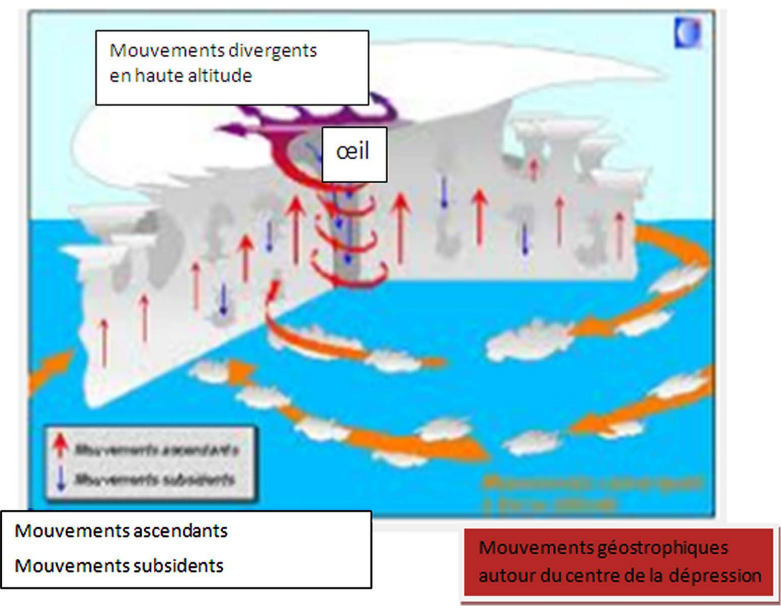

(c)

Figures 3. Schematic representations of Hurricanes and related cloud or electrical systems. Hurricanes' new-formed clouds are gray while old-formed clouds are blue.

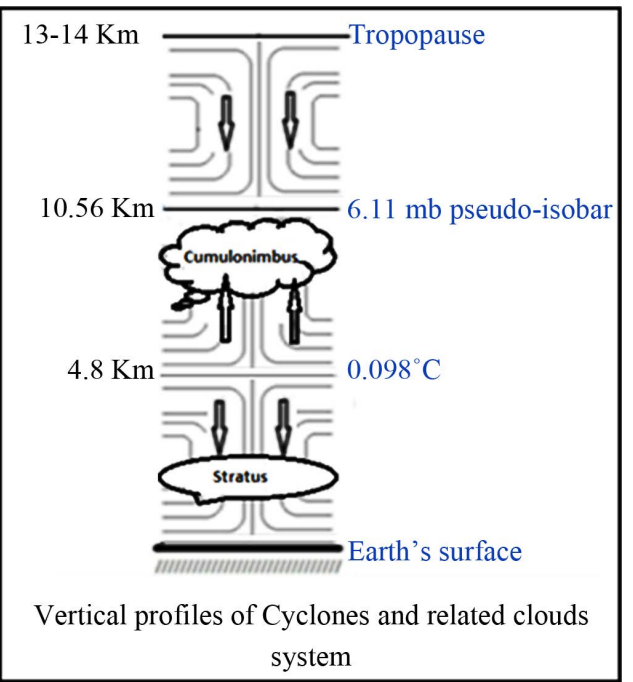

(a)

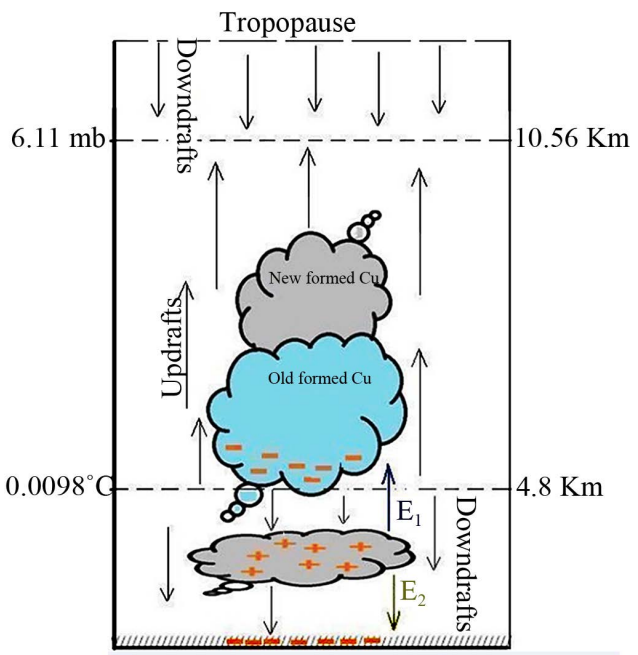

(b)

Figures 4. Schematic representations of Cyclones and related cloud or electrical systems. Cyclones' new-formed clouds are gray while old-formed clouds are blue.

the frictional force and the tidal force are (from surface of the earth to tropopause) negligible compared to the Coriolis and pressure-gradient forces. When pressure gradient and Coriolis forces are the only two factors acting, geostrophic winds (rotative in the Northern hemisphere and contra-rotative in the Southern hemisphere) immediately take place (Figures 5) within deep and passive convections. The impact of hurricanes footprint (less than a dozen kilometers in diameter) is much lower than that of cyclones (several tens of kilometers in diameter).

d) Thermodynamic or chemical constraints on rain

Clouds are physical systems consisting of water particles. Even if the volume of a water particle is large; as long as its density $r_{w}(z)$ remains less than that of the surrounding air $r_{A}(z)$, the particle remain suspended in the 


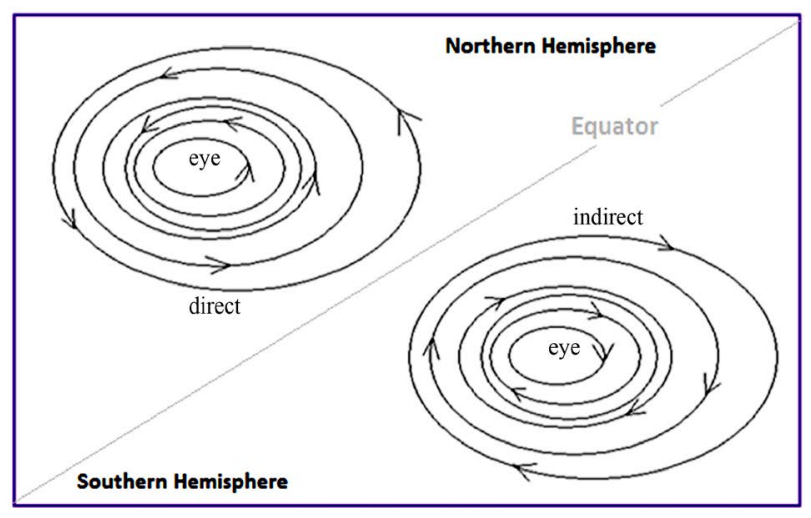

Figures 5. Schematic representations of Cyclones and Tornadoes geostrophic balance winds.

air. For causing its fall: the water particle must be contaminated with a soluble chemical substance having density greater than $r_{A}($ at $z=0)$. Natural Contaminations are performed with carbon dioxide; sulfur emitted by volcanoes or sprays emitted by the oceans, etc. Acid rains reflect this thermodynamic constraint. Clouds separate themselves with favorably contaminated water particles and that give rise to rainfall or snowfall.

\subsection{Relationship between Cloud Formation's Additional Green House and Tornadoes}

Considering the molecular scale, our model (Figure 6) based on Mbane Biouele formula (2009) suggests blocking of hot updrafts by hot down drafts which means installation of an additional greenhouse that triggers the superposition of cloud formation latent heat with earth's surface radiate heat $R_{T}\left(R_{T}=\varepsilon_{S} \sigma T_{S}^{4}\right)$. This is consistent with based observations and explains high surface temperatures that accompany the formation of clouds in the sunny sky. Furthermore, Tornadoes look like little-bombs triggered by cloud's additional greenhouse. Indeed, Rayleigh and Reynolds numbers of passive convection motions depend mainly on air parcel temperature and humidity. Within additional greenhouse, temperature and water vapor increase exponentially and finally (as atmosphere is a dissipative system) trigger violent adiabatic expansion or tornadoes (as Rayleigh and Reynolds numbers become higher and generate turbulent motions). Thermodynamic processes that govern the formation of tornadoes or hurricane (Figures 3(a) and (b)) are well known now.

\subsection{Appropriate and Unique Representation of Earth's Atmosphere General Circulation}

According to our previous study [9], the presence of water substance in all three states in the earth's atmosphere gives to troposphere the exclusivity of a general circulation consists of three groups of passive convective cells
(Hadley, Ferrel, and Polar) on either side of the ITCZ (Inter Tropical Convergence Zone). These cells (H, F, and P) take place within areas bounded by both tropopause and the two equal level surfaces of water vapor and temperature rating respectively at $6.11 \mathrm{mb}$ and $0.0098^{\circ} \mathrm{C}$ as suggested in Figure 7.

\section{Conclusions and Comments}

All natural meteorological events including hurricanes and cyclones can be traced to the manner in which the energy from the sun is received over different parts of the earth. Since the troposphere is a medium in which mass motions are easily started, convection is found to be one of the chief ways in which heat is transferred there. This

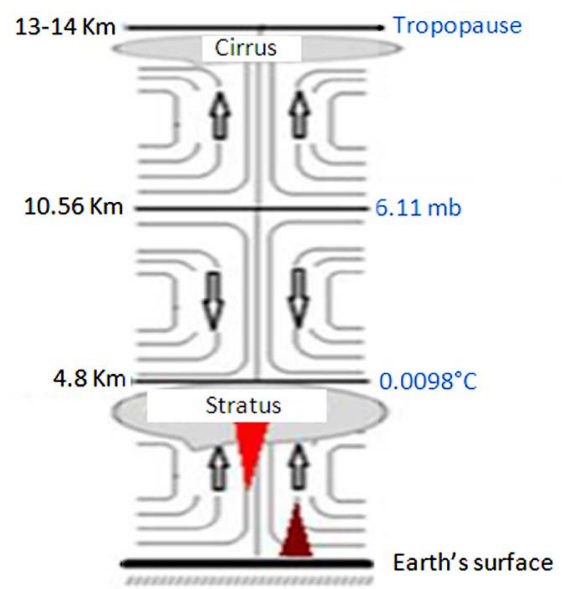

Mbane Biouele formula (2009) suggests blocking of hot updrafts by hot down drafts: then installation of an additional greenhouse which triggers the superposition of cloud formation latent heat (red color) with earth's radiating heat $e_{5} \mathrm{~s}^{4}$ (brown color). That explains high surface temperatures that accompany the formation of clouds in the sunny sky (or in extreme cases: the formation of tornadoes)

Figure 6. Clouds formation additional green house.

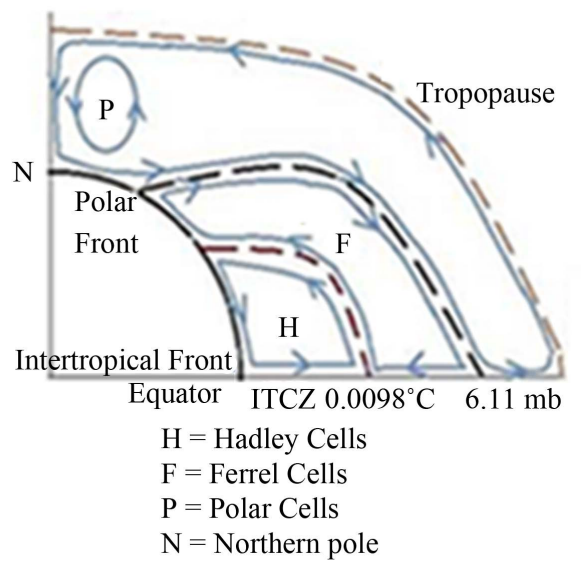

Streamlines of Earth's Atmosphere General Circulation

Figure 7. Schematic representation of the General Circulation, derived from Mbane Biouele formula (2009) and related corollaries. 
transfer may be accomplished either by vertical or by horizontal motions. According to our results: warmer disturbances that occur in lower-troposphere are dissipated by a typical mass motion usually called Hurricanes (or tornadoes) while cooler disturbances that occur in mid-troposphere are dissipated by another typical mass motion called Cyclones. Thermal and Kinematic profiles of atmosphere disturbances that have both a large and a strong destructive power clearly indicate that these terrible events for men should not be viewed with fatalism. Viewing the urgency to perform our knowledge of tornadoes or cyclones and erasing our great fear of these events, theatricalization of meteorology becomes something unacceptable. Our research field (meteorology in this case) can be popularized while remaining serious about what is presented to those who consume it. We hope that one day the weak-points of hurricanes and tornadoes will be exploited to neutralize them. e.g., although costly, procedures to extinguish the huge forest fires are developed and used successfully. It is possible, we reflect deeply on this issue, that the neutralization of tornadoes requires less effort than those made to forest fires.

\section{Acknowledgements}

Author of this paper gratefully acknowledge the following University Institutions and Professors: 1) The University of California in Los Angeles (USA) who kindly provided a geospatial measurement station called "AMBER MAGNETOMETER" to the University of Yaoundé I (Cameroon); The Air Force Research Laboratory (Boston University), which has equipped the University of Yaoundé I with two geospatial measurement stations called "SCINDA SENSORS" and The University of Yaoundé I which offered modern facilities with Internet Connection, to accommodate both stations. 2) Professors: Mark B. Moldwin (Atmospheric-Oceanic and space Sciences Research Laboratory, University of Michigan (USA)) and
Endawoke Yizengaw (Institute for Scientific Research (USA)), for their very kind collaboration.

\section{REFERENCES}

[1] B. Couanang Siebatcheu, C. Mbane Biouele and J. S. Eyebe Fouda, "Atmosphere Dynamic Balance Model (ADBModel) and Related Troposphere General Circulations' Cells behind the Formation of Tropical Monsoons," Scholars Research Library-Archives of Physics Research, Vol. 3, No. 2, 2012, pp. 93-100.

[2] C. Mbane Biouele, "Hurricanes and Cyclones Kinematics and Thermodynamics Based on Clausius-Clapeyron Relation Derived in 1832," International Journal of Physical Sciences, Vol. 8, No. 23, 2013, pp. 1284-1290.

[3] C. Mbane Biouele, E. Yizengaw, M. B. Moldwin and G. Cautenet, "Impacts of Thermoelastic Properties of Saturated Water Vapor on Tropical Depressions Thermodynamics and Dynamics," Scholars Research Library-Archives of Physics Research, Vol. 2, No. 4, 2011, pp. 24-33.

[4] C. Mbane Biouele, "Vertical Profiles of Winds and Electric Fields Triggered by Tropical Storms-Under the Hydrodynamic Concept of Air Particle”, International Journal of Physical Sciences, Vol. 4, No. 4, 2009, pp. 242246.

[5] C. Mbane Biouele, "Physics of Atmosphere Dynamic or Electric Balance Processes Such as Thunderclouds and Related Lighting Flashes,” Geosciences, Vol. 2, No. 1, 2012, pp. 6-10.

[6] H. R. Byers, “General Meteorology,” McGraw-Hill Book Company, INC., 1959, 540 p.

[7] G. K. Batchelor, “An Introduction to Fluid Dynamics,” Cambridge University Press, 1967, 496 p.

[8] C. A. Riegel, "Fundamentals of Atmospheric Dynamics and Thermodynamics," World Scientific Publishing Co. Pte. Ltd., 1992, 512 p.

[9] C. Mbane Biouele, "Application of Clausius-Clapeyron Relation (1832) and Carnot Principle (1824) to Earth's Atmosphere Tricellular Circulation," Atmospheric and Climate Sciences, Vol. 4, No. 1, 2014, pp. 1-6. 